\title{
CCD-Type Multi-Ion Image Sensor with Two Kinds of Plasticized Poly(vinyl chloride) Membranes
}

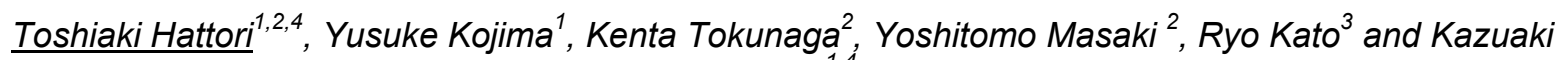 \\ Sawada ${ }^{1,4}$. \\ 1 Department of Electrical and Electronic Information Engineering, 2 Department of Materials Science, \\ 3 Cooperative Research Facility Center, Toyohashi University of Technology, Toyohashi, JAPAN. \\ 4 Core Research for Evolutional Science and Technology (CREST), JAPAN. \\ Corresponding author's Email address, thattori@ee.tut.ac.jp
}

\begin{abstract}
A charge coupled device (CCD)-type multi-ion image sensor for sodium ion and potassium ion was fabricated. Two kinds of plasticized poly(vinyl chloride) (PVC) membranes contained different ionophores were separately prepared on the semiconductor image sensor. The membrane preparations were occurred by a conventional casting method with hand painting, and inkjet painting with a laboratory inkjet printing apparatus. Since the casting solvent of tetrahydrofuran (THF) has low viscosity and high osmosis, a pre-constructing PVC membrane was easily invaded by the next THF casting solution. Then a partition wall was constructed in the center of the sensor region in order to prevent two membranes from contacting. The hand painting was fast and easy without special equipment, but it was hard to control each sensing area finely. The inkjet painting can finely prepare each membrane to control the painting area. In inkjet painting, however, THF was inappropriate as its printing solvent because of high volatility. The mixture solvent of THF and cyclohexanone (CHN) was suitable. Both CCD-type multi-ion image sensors prepared by different ways displayed that each membrane apparently responded to only sodium ion or only potassium ion, according to their selectivity coefficients. Sodium and potassium ions can be simultaneously monitored on the CCD-type multi-ion image sensor.
\end{abstract}

Key words: CCD-type multi-ion image sensor, plasticized PVC membrane, potassium ion, sodium ion, hand painting, inkjet method.

\section{Introduction}

Semiconductor chips arrayed a lot of microsensors are useful to observe biological activity of cells and tissues. A high-resolution multitransistor array can record electrical field potentials in cultured brain slices [1]. The sensor array was excellent in obtaining very fast signals of neural activity noninvasively. In order to widely-spread two-dimensional array semiconductor sensors into chemical and biological observations, quantitative data such as the concentration of analyte ion are required. Sawada et al. has developed a $\mathrm{pH}$ image sensor that was based on charge coupled device (CCD) and complementary metal oxide semiconductor technologies [2]. The image sensor applied to measure diverse ions besides hydrogen ion. We had developed several CCDtype ion image sensors with plasticized PVC membranes containing ionophores. The potassium ion image sensor dynamically monitored the potassium ion leakage from a 3.3
$\mathrm{M} \mathrm{KCl}$ solution $\mathrm{Ag} / \mathrm{AgCl}$ reference electrode [3]. The sodium ion image sensor can simply and easily monitor the ion-exchange reactions of a single Na-type cation-exchange resin bead [4]. It was displayed that the kinetic progress of $\mathrm{Na}^{+}-\mathrm{Ba}^{2+}$ ion-exchange was faster than that of $\mathrm{Na}^{+}-\mathrm{Ca}^{2+}$ ion-exchange. Thus, the usefulness was confirmed as a monitor of the chemical reaction.

In this study we demonstrate multi-ion image sensors with two kinds of plasticized poly(vinyl chloride) membranes. Since the sensing area of the semiconductor image sensor was small (a square about $4 \mathrm{~mm}$ ), preparation of different membranes on the same image sensor required skillful techniques. First, we used a conventional casting method with hand painting to prepare divided sensing regions. The way was fast and easy without special equipment, but it was hard to control each sensing area finely. Next, we applied an inkjet printing method to preparing two kinds of membranes. THF has low viscosity and high 
osmosis, and was inadequate as the inkjet solvent. Several solvents and mixture solvents which can dissolve the membrane content were examined to adjust them to the inkjet solvent. The mixture of THF and $\mathrm{CHN}$ was suitable to the inkjet solvent, and each painting area was controlled.

\section{Experimental}

\section{Reagents}

Bis[(12-crown-4)-methyl]-2-dodecyl-2-methyl malonate (bis(12-crown-4)), Bis-[(benzo-15crown-5)-4-methyl]pimelate (15-crown-5), sodium tetrakis[3,5-bis(trifluoromethyl)phenyl]borate (Na-TFTB) were purchased from Dojindo Laboratories, Japan. PSS-Methacryl substituted. Cage mixture, $n=8,10,12$ (PSS), PVC (average molecular weight; 8200), tris(hydroxymethyl)-aminomethane (Tris), potassium tetrakis(4-chloro-phenyl)borateand (K-TCPB) were purchased from Sigma-Aldrich. Dioctyl phthalate (DOP) and other reagents were prepared from analytical reagent grade chemicals. Metal ion solutions were prepared daily by dilution from $1 \mathrm{M}$ stock solutions of metal chlorides.

CCD-type ion image sensor

Fig. 1 shows the apparatus of CCD-type ion image sensor. The sensor possessed 1024 sensing pixels that formed a two-dimensional array of $32 \times 32$. The area of the sensor was $4.16 \times 4.16 \mathrm{~mm}^{2}$. Each pixel was a square of $130 \mu \mathrm{m}$, and the sensing region was about a square of $40 \mu \mathrm{m}$. The electrochemical cell consisted of reference $\mathrm{Ag} / \mathrm{AgCl}$ electrode / sample solution / plasticized PVC membrane / semiconductor sensor $\left(\mathrm{Si}_{3} \mathrm{~N}_{4} /\right.$ thin $\left.\mathrm{SiO}_{2} / \mathrm{p}-\mathrm{Si}\right)$. The potential of the PVC membrane altered the depth of the potential well formed in the surface of the $\mathrm{p}-\mathrm{Si}$ under the thin $\mathrm{SiO}_{2}$ layer. The potential well depth was measured by the CCD as the amount of charge added from an input source. The output signal was processed through an external analog-digital converter and recorded by a computer. The measurement system consisted of a CCD sensor, a pulse generator, and a computer. Each pixel had a selection transistor, which could turn on the select signal. The time interval for one pixel from the first reset to readout was $333 \mu$ s. Computer software converted the signals into a moving image at five frames per second. The ion images were measured in a dark box to avoid the influence of electric charge produced from the photosensitivity of $\mathrm{p}$-Si.

The output potential was not directly equal to the membrane potential. The output potential arose from the capacities of the sensing area and a floating diffusion (FD) in one pixel, and a transfer gain of the source follower circuit. The ideal output potential was $31.5 \mathrm{mV}$ decade ${ }^{-1}$, which was determined by multiplying the total output gain in the semiconductor by the ideal membrane potential change, $59.1 \mathrm{mV}$ decade $^{-1}$ when the membrane potential followed a Nernstian response. The total output gain was obtained by multiplying a transfer gain by the source follower gain. The transfer gain was a ratio of the electric capacity of $\mathrm{p}-\mathrm{Si}$ to the electric capacity of the FD.

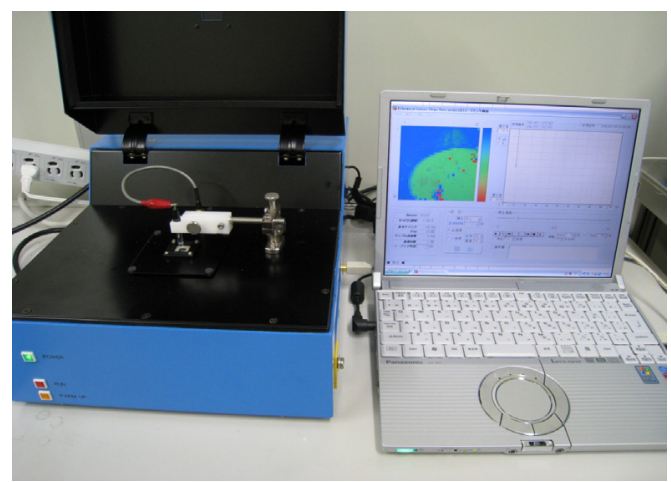

Fig.1: Photograph of whole apparatus including CCD-type ion image sensor and a note-type personal computer.

Tab. 1: Contents of plasticized PVC membranes and partition wall

\begin{tabular}{|l|c|c|c|}
\hline Materials & Na-membrane & K-membrane & Partition Wall \\
\hline PVC & $44 \%$ & $44 \%$ & $90 \%$ \\
\hline PSS & $30 \%$ & $30 \%$ & $10 \%$ \\
\hline DOP & $22 \%$ & $22 \%$ & - \\
\hline lonophore & $2.7 \%$ of $12-c r o w n-4$ & $2.7 \%$ of $15-c r o w n-5$ & - \\
\hline lon exchanger & $1.3 \%$ of Na-TFTB & $1.3 \%$ of K-TCPB & - \\
\hline
\end{tabular}


Preparation of plasticized PVC membranes

Each plasticized membrane was prepared by dissolving the components listed in Tab. 1 in $5.0 \mathrm{~mL}$ of THF or mixture solution of THF and $\mathrm{CHN}$. On the hand painting, the partition wall was prepared by a pile painting using a fine brush. Thus THF solution of the partition wall content (Tab.1) was painted at the center in the image sensor. After the partition wall was constructed and dried, the sodium ion sensitive membrane (Na-membrane content in Tab. 1) and potassium sensitive membrane (Kmembrane content in Tab. 1) were prepared by a conventional drop-casting.

\section{Results and Discussion}

Hand painting

It was difficult to prepare two kinds of plasticized PVC membrane directly by ordinary casting method. The casting solvent of THF has low viscosity and high osmosis, and then, a preconstructing PVC membrane was easily invaded by the next THF casting solution. Therefore we made a partition wall by a hand painting in order to prevent two membranes from contacting. The proper content of each membrane was used as previously reported $[4,5]$. The potential properties to each ion concentration of Na-membrane and $\mathrm{K}$ membrane are shown in Fig. 2 and 3. The potential slope of Na-membrane was $29.5 \mathrm{mV} /$ decade to sodium ion. The potential slope of K-membrane was $31.0 \mathrm{mV} /$ decade to potassium ion. The slopes indicated both membranes had Nernstian response. The $\mathrm{Na}$ membrane indicated that the selectivity of sodium ion was higher than that of potassium ion. The selectivity of K-membrane was vice versa. The sensor images in various concentrations of sodium ion and potassium ion are shown in Fig. 4. The right hand of $\mathrm{Na}$ membrane apparently responds to only sodium ion. The left hand of K-membrane apparently responds to only potassium ion. As the partition wall, there is unresponsive region (pixels) at the center part. The hand painting, however, was hard to control each sensing region and cannot reduce usefulness area of the partition wall.

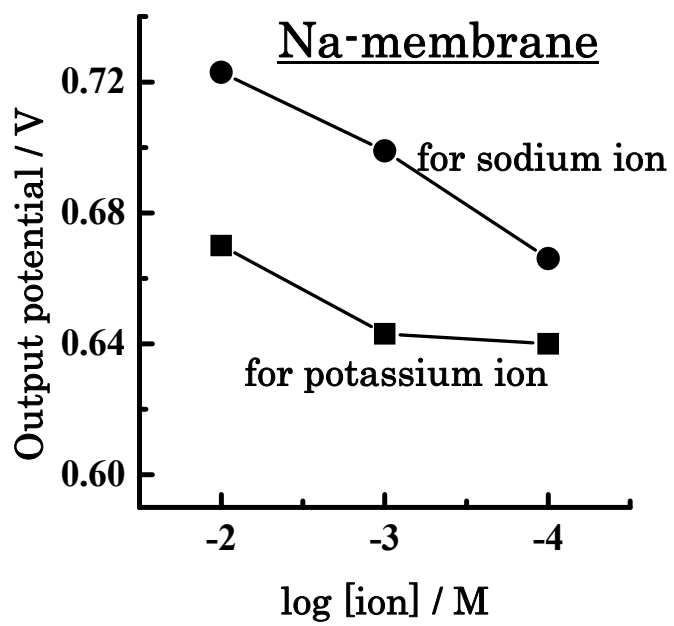

Fig. 2: Potential response of sodium ion sensitive membrane for concentration change of potassium ion and sodium ion.

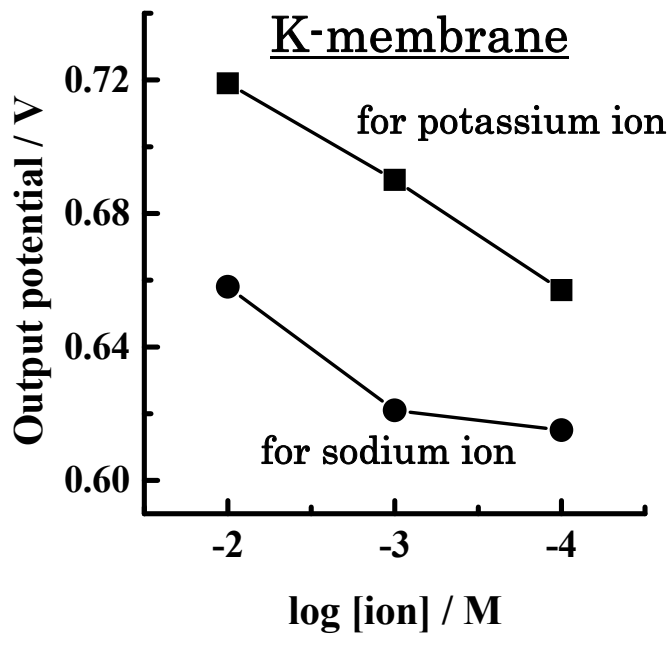

Fig. 3: Potential response of potassium ion sensitive membrane for concentration change of potassium ion and sodium ion.
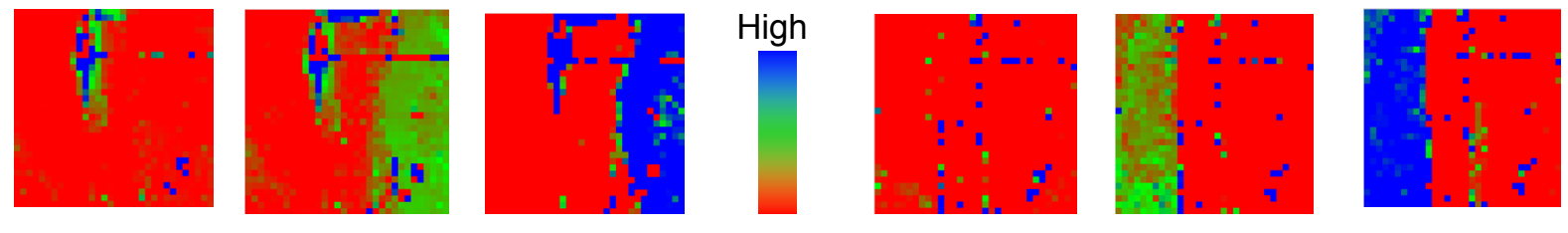

$10^{-4} \mathrm{M} \mathrm{NaCl}$

$10^{-3} \mathrm{M} \mathrm{NaCl}$

$10^{-2} \mathrm{M} \mathrm{NaCl}$

Low
$10^{-3} \mathrm{M} \mathrm{KCl}$

$10^{-2} \mathrm{M} \mathrm{KCl}$

Fig. 4: Images of a conventional casting Multi-ion images sensor for various concentrations of sodium and potassium ions. Left: K-membrane. Right: Na-membrane. 
Inkjet Painting

An inkjet printing apparatus (IJK-200T, Microjet Co. Ltd, Japan) was used for preparation of two kinds of membranes and the partition wall. The apparatus was a piezoelectric dot injekt, and a dot volume was several tens of $\mathrm{pL}$. The painting with a blank space between dots was not suitable because of the lack of durability of membrane. Thus the image sensor should be entirely covered with plasticized PVC.

As the inkjet solvent, $\mathrm{CHN}$ which solved the membrane content slowly has higher viscosity and slower volatility than THF. However, dry of dots with only CHN took many times. Therefore, the mixture solvent of THF and $\mathrm{CHN}$ was useful. The Na-membrane and K-membrane were the same contents as listed in Tab. 1.

Figure 5 shows transient response images of an inkjet printing multi-ion image sensor at each time, after $10^{-2} \mathrm{M} \mathrm{KCl}$ solution was added into $10^{-4} \mathrm{M} \mathrm{KCl}$ solution. The upper region painted with K-membrane only responds to potassium

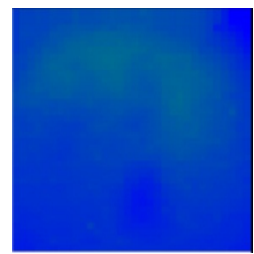

$0 \mathrm{~s}$

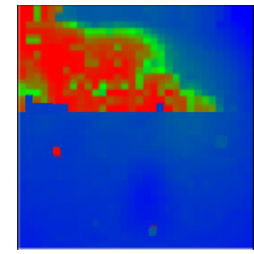

$0.2 \mathrm{~s}$

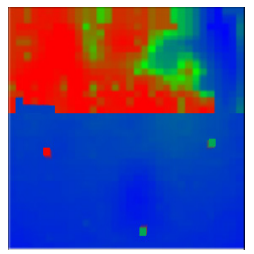

$0.4 \mathrm{~s}$

ion. The lower region painted Na-membrane was unresponsive to potassium ion. The potential slope of K-membrane by the inkjet method was $29.3 \mathrm{mV} /$ decade which was almost Nernstian response.

Figure 6 shows transient response images of an inkjet printing multi-ion image sensor at each time, after $10^{-2} \mathrm{M} \mathrm{NaCl}$ solution was added into $10^{-4} \mathrm{M} \mathrm{NaCl}$ solution. The lower region painted with $\mathrm{Na}$-membrane only responds to sodium ion. The upper region painted with K-membrane was unresponsive to sodium ion. The potential slope of K-membrane by the inkjet method was $22.6 \mathrm{mV} /$ decade, and indicated a sub-Nernstian response.

In conclusion, a multi-ion image sensor with two kinds of plasticized PVC membranes was developed. Each membrane apparently responded to only sodium ion or only potassium ion. The inkjet method can finely control the painting area of each membrane.

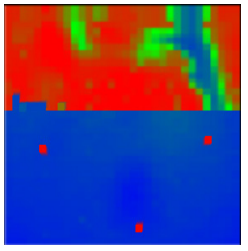

$0.8 \mathrm{~s}$

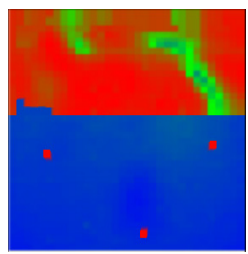

$1.4 \mathrm{~s}$

Fig. 5: Transient response images of an inkjet printing multi-ion image sensor after $10^{-2} \mathrm{M} \mathrm{KCl}$ solution was added into $10^{-4} \mathrm{M} \mathrm{KCl}$. Upper: K-membrane. Lower: Na-membrane.

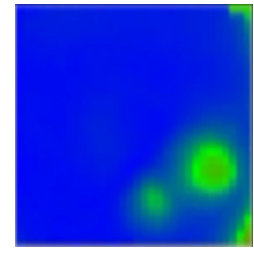

$0 \mathrm{~s}$

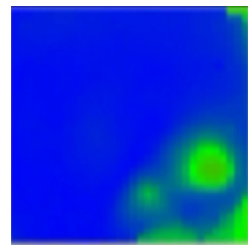

$0.2 \mathrm{~s}$

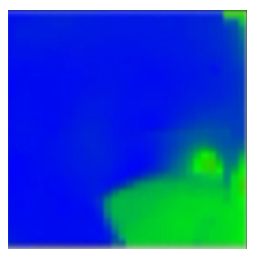

$0.4 \mathrm{~s}$

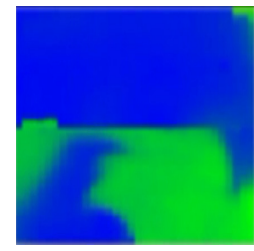

$0.8 \mathrm{~s}$

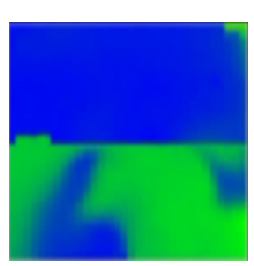

$1.6 \mathrm{~s}$
Low

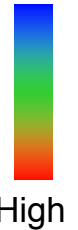

High

Fig. 6: Transient response images of an inkjet printing multi-ion image sensor after $10^{-2} \mathrm{M} \mathrm{NaCl}$ solution was added into $10^{-4} \mathrm{M} \mathrm{NaCl}$. Upper: K-membrane. Lower: Na-membrane.

\section{Acknowledgements}

This work was supported in part by a Grant in Aid for Scientific Research (No. 23615005) from Ministry of Education, Culture, Sports, Science and Technology, Japan.

\section{References}

[1] Hutzler, M., A. Lambacher, B. Eversmann, M. Jenkner, R. Thewes, and P. Fromherz., J. Neurophysiol, 96, 1638-1645, 2006.

Doi:10.1152/jn.00347.2006.
[2] T. Hizawa, K. Sawada, H. Takao, and M. Ishida, Sens. Actuators B, 117, 509-515 (2007). Doi:10.1016/j.snb.2006.01.056.

[3] T. Hattori, Y. Masaki, K. Atsumi, R. Kato, K. Sawada, Anal. Sci., 26, 1039-1045 (2010). Doi:10.2116/analsci.26.1039

[4] T. Hattori, Y. Masaki, S. Mori, D. Miyamoto, R. Kato, K. Sawada, Electroanalysis, 24, 114-120 (2012). Doi:10.1002/elan.201100442. 